\title{
Achievement of Small Target Detection for Sea Ship Based on CFAR-DBN
}

\author{
LiBing Yuan, ${ }^{1}$ XueLi Chi, ${ }^{1}$ and Hui Wei $\mathbb{D}^{2}$ \\ ${ }^{1}$ Sanya Aviation Tourism Vocational College, Sanya 572022, Hainan, China \\ ${ }^{2}$ College of Electronic and Information Engineering, Henan Institute of Technology, Xinxiang 453000, Henan, China
}

Correspondence should be addressed to Hui Wei; dxweihui@hait.edu.cn

Received 15 December 2021; Revised 15 January 2022; Accepted 1 February 2022; Published 18 February 2022

Academic Editor: Zhiguo Qu

Copyright (C) 2022 LiBing Yuan et al. This is an open access article distributed under the Creative Commons Attribution License, which permits unrestricted use, distribution, and reproduction in any medium, provided the original work is properly cited.

With the rapid development of marine exploration and marine transportation, the activities of marine ships are becoming more and more frequent. Accurate and rapid detection of the position of marine ships has very important practical and strategic significance. SAR has the characteristics of all-weather detection. It is an important means of ship detection. Aiming at the problem of fuzzy interference in sea surface ship SAR image detection, a small target image detection algorithm based on constant false alarm rate (CFAR) and depth belief network (DBN) is proposed. Firstly, according to the traditional CFAR detection principle, the whole image to be detected is detected by CFAR globally, and the index matrix is obtained, so as to improve the ship detection speed. Secondly, the output data of the hidden layer of the last layer of DBN are used as the input data of SVM, and the trained DBN model is applied to local detection, so as to improve the accuracy and robustness of ship detection. Finally, the algorithm combining CFAR and DBN is trained and applied in ship detection. Experimental results show that the accuracy of the proposed algorithm under fuzzy interference is better than that of traditional CFAR, BPNN, Fast R-CNN, and SSD512 algorithms, which proves that the robustness of the combined algorithm is significantly improved.

\section{Introduction}

Ship is an indispensable means of transportation for sea transportation and exploitation of seabed resources. With the rapid development of maritime economy and the increasing number of ships on the sea, the safety requirements of maritime transportation are becoming higher and higher. Therefore, it is an urgent problem to accurately detect the position of naval vessels in coastal areas [1-5]. Therefore, the ship detection and recognition technology is very important, and the need to use information means to improve the supervision ability and confrontation ability is increasing.

With the rapid development of synthetic aperture radar (SAR) imaging technology [6-8], it is playing an increasingly important role in the civil and military fields. This technology can realize the accurate detection, real-time monitoring, and state capture of hull targets. SAR is a sensor using active microwave remote-sensing technology, which can complete the task of detecting targets in all-weather, multiangle, and multidimensional fields in the application of marine resources' supervision [9-11]. With the maturity of SAR technology, the coordination mechanism between SAR system and signal processing algorithm has attracted extensive attention. Researchers can obtain more semantic information on the content of the target information, including different bands, polarization mode, and resolution. In addition, SAR images can provide complete data information for researchers to mine and explore the rich multidimensional information, thus improving the ability of target detection and recognition more effectively.

CFAR algorithm is currently the most widely used among many target detection algorithms and has the best effect. The core idea is to establish the sea clutter distribution model based on local area sea clutter data and draw the probability density curve of the distribution model. Then, the target pixel segmentation threshold is calculated by the false alarm rate, and finally, the high gray value target in the SAR image is detected by the threshold. On the basis of 
classical CFAR technology, scientists have spent a lot of efforts to improve the CFAR detection algorithm from different aspects to improve its detection performance. Tao et al. [12] proposed a segmented CFAR algorithm, which is very suitable for target detection in local regions. Palamà et al. [13] proposed a change index CFAR algorithm from an adaptive perspective, which can dynamically select the unit average according to the set assumed conditions. Jiang et al. [14] proposed a CFAR with adaptive selection of pixel sets, which performs well in uniform clutter environments. However, the above detection algorithms are all single CFAR detection algorithm, each of which has its own advantages and disadvantages, as well as applicable conditions. A single CFAR detection algorithm performs well in the case of uniform clutter background, but it lacks high accuracy and robustness for the nonuniform background (fuzzy interference) with clutter interference.

As a hot technology with rapid development in the field of artificial intelligence in recent years, deep learning has been widely used in various industries. Deep learning comprehensively learns the abstract information from low level to high level through nonlinear elements [15-17], which reduces the complex preprocessing in the early stage of artificial intelligence and improves the efficiency of target detection and recognition. At present, the research on ship target detection in SAR images based on depth learning has become a hot topic in the application of related fields. Researchers are dedicated to obtaining high-resolution, high-robustness ship target detection techniques in a wider range of scenarios, thus solving the real-time and complexity problems in real-world environments. Wang et al. [18] proposed an improved region-based total volume network (R-FCN) detection method, which extracts the features of ship targets and performs mixed multiscale processing, and achieved good detection results. Aiming at the problem that small targets are difficult to locate, Chen et al. [19] proposed a depth learning detection method combined with attention mechanism, which obtained the region of interest by automatically learning the attention mechanism feature map. Among many algorithms, how to quickly select the better algorithm to adapt to the corresponding scene is also an urgent problem to be solved in this field.

Deep belief network (DBN) is a probabilistic machine learning model for multilevel neural networks [20-22]. DBN reconstructs the input data through a layer-by-layer greedy learning algorithm and then globally fine-tunes the initialized network parameters through a supervised algorithm. The features learned and extracted from the raw data by DBN can achieve a higher-level expression description of the raw data. This learning method overcomes some problems of previous neural network structures, such as local optimum and unstable training time caused by initialization parameters. DBN is widely used in image recognition classification, face recognition, handwritten font recognition, and other fields.

Therefore, this paper attempts to combine CFAR algorithm with $\mathrm{DBN}$ to solve the problem of nonuniform background (fuzzy interference) in clutter interference. The purpose of this research is to overcome the influence of fuzzy interference on the detection of small targets on the surface of the ship and improve the accuracy and robustness of target detection. First, the target index matrix is obtained by the global detection in CFAR algorithm. Then, the detected image is intercepted with the target index as the center, and the trained DBN model in DBN-SVM algorithm is used to detect it. Finally, all detection results are combined to obtain a final detection result.

\section{SAR Imaging Ambiguity Principle}

The imaging method of radar is active imaging. Electromagnetic wave is transmitted at a certain time interval during that continuous movement of the imaging device. After the electromagnetic wave irradiates the object, the reflection is formed, and the echo information is transmitted back to the imaging device. Then, through a series of algorithms to process the echo information, the imaging process is completed. In this process, SAR signals are usually stored as two-dimensional arrays. SAR echoes in the twodimensional plane can have two dimensions: distance and azimuth. If the sampling does not match, it will form a blur, reduce the image quality, and even affect the target detection and judgment. Therefore, we need to study the characteristics of two forms of blur, so as to provide the necessary conditions for verifying the robustness of the algorithm.

2.1. Azimuth Ambiguity. SAR uses pulse repetition frequency (PRF) [23] to sample the echo Doppler signal to obtain the echo information. The corresponding Doppler bandwidth matched filter is within the main bandwidth. When the PRF is high, it will not be affected by the side lobe signal basically. However, when the PRF is undersampled, in the azimuth echo spectrum, other nontarget signals will enter the matched filter, and thus affect the target sampling in the main lobe. Nontarget signals are aliased with main signals to form azimuthal ambiguity. The aliasing phenomenon occurring in the side lobe can lead to the generation of false targets [24]. Azimuth ambiguity diagram is shown in Figure 1.

2.2. Distance Ambiguity. In addition to azimuth ambiguity, there is another common way of ambiguity, that is, distance ambiguity. Compared with azimuth ambiguity, distance ambiguity is mainly caused by high PRF. The higher the pulse repetition frequency, the smaller the time interval between pulses. Specifically, it shows the time delay between the target echo signal in a certain area and the previous pulse signal. Overlap with the echo time delay in the mapping band of the main lobe causes interference [25]. Figure 2 is a schematic diagram of distance ambiguity, in which distance ambiguity is formed in the echo region where time delay occurs.

\section{Ship Small Target Detection Algorithm Based on CFAR-DBN Under Fuzzy Interference}

3.1. Global CFAR Algorithm. At present, CFAR detection algorithm is a more classical method in SAR image target 


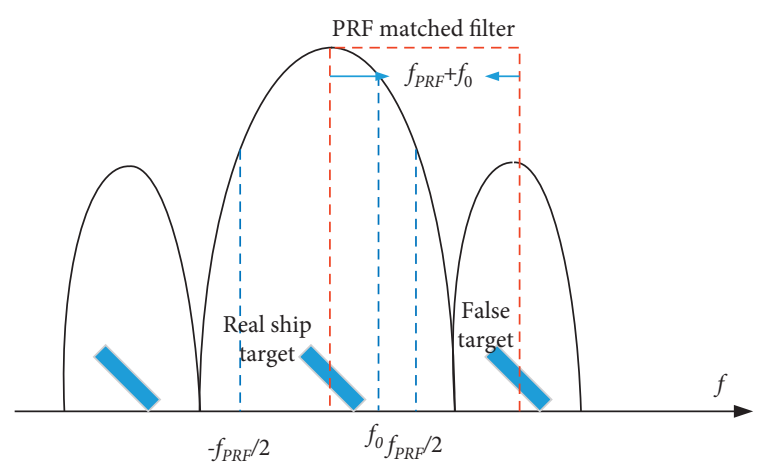

FIgURE 1: Schematic diagram of azimuth ambiguity.

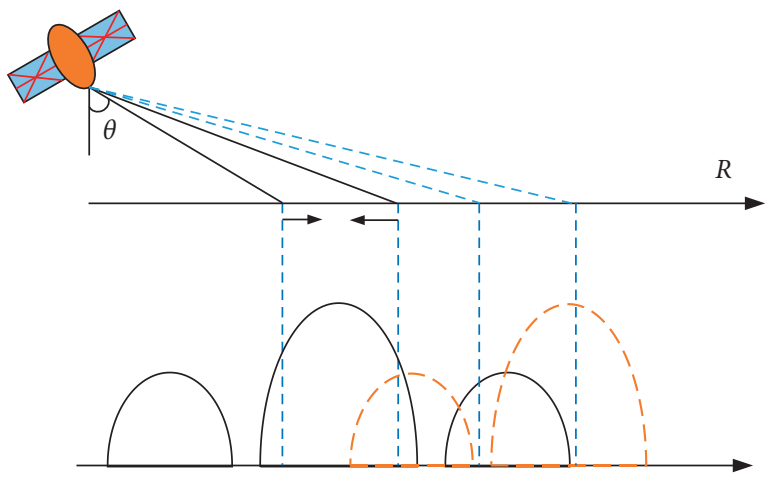

Figure 2: Schematic diagram of distance ambiguity.

detection. The CFAR algorithm compares the pixel gray levels of possible targets with the thresholds determined from the surrounding background. This paper uses this algorithm in global detection.

First, the value of CFAR cannot be set accurately. Therefore, for the false alarm rate of global detection, the fuzzy estimation value is used as the initial value. The calculation method for estimating the false alarm rate $P_{f a}$ of the whole SAR image is as follows:

$$
P_{f a} \approx \frac{A_{\text {ship }}}{A_{\text {sar }}}
$$

where $A_{\text {ship }}$ represent pixel area of all vessels in the image and $A_{\text {sar }}$ represent the pixel area of the entire SAR image.

By histogram statistics of the gray values of all clutter points in SAR images [26], the probability distribution of histogram can be obtained as $f(x)$. Set the global CFAR value $\left(P_{f a}\right)$ and solve formula (2) to get the global threshold $T_{\text {low. }}$. If the gray value $T$ of the pixel in SAR image is greater than the threshold $T_{\text {low }}$, it is considered to be abnormal in the surrounding clutter, and it is judged as the ship target, which is denoted as $T_{1}$; otherwise, it is judged as the ocean clutter, and the judgment rule is shown in formula (3):

$$
\begin{aligned}
& P_{f a}=\int_{T}^{\infty} f(x) \mathrm{d} x, \\
& T \begin{cases}>T_{\text {low }}, & \text { ship target, } \\
<T_{\text {low }}, & \text { ocean clutter. }\end{cases}
\end{aligned}
$$

After the global threshold $T_{\text {low }}$ is calculated, it is compared with the gray values of all pixels in the image. If the gray value of the pixel is greater than $T_{\text {low }}$, set it to 1 . Otherwise, set to 0 . After all the pixels are compared, the index matrix is obtained.

Global target detection adopts CFAR detection based on $\mathrm{K}$ distribution, and the probability density function (PDF) of $\mathrm{K}$ distribution is

$$
P(x)=\frac{2}{x \Gamma(v) \Gamma(n)}\left(\frac{n v x}{\mu}\right)^{(n+v / 2)} K_{v-n}\left(2 \sqrt{\frac{n v x}{\mu}}\right),
$$

where $v$ represents the state of the function, $\mu$ represents the dimension of the function, $n$ represents the estimate of the function to the whole SAR image, and $K_{v-n}(\cdot)$ is a Bessel function of order $(v-n)$. When the clutter model is $K$ distributed, firstly, transform the image data, and we can obtain

$P_{f a}=\int_{T}^{\infty} p(y) \mathrm{d} y=1-F(t)=\frac{2}{\Gamma(v)}\left(\frac{v}{\mu}\right)^{(v / 2)} T^{v} K_{v}\left(2 \sqrt{\frac{v}{\mu}} T\right)$.

The gray level of the target pixel is compared with the threshold obtained by the surrounding background. The false alarm rate is usually obtained through experience, and the threshold of statistical distribution can be obtained according to relevant formulas. The traditional CFAR method presets the false alarm rate. We can judge whether the pixel to be detected is the target point by CFAR algorithm. According to the prior knowledge of the target, we select the appropriate sliding window to detect the whole SAR image point by point. Sliding windows are divided into hollow and solid. The sliding window in the solid window is consistent with the target size, while the sliding window in the hollow window is larger than the target size. The size of the solid sliding window is equal to that of the target, but due to the principle of SAR imaging, the surroundings of the target and the background will interfere with each other, which will have a certain influence on the judgment of the target. In order to eliminate these influences, we remove these pixels, so we choose the background points with a certain distance from the target as the judgment data.

With the center of the target point in the index matrix, three windows are established: target window $(T)$, protection window $(S)$, and background window $(G)$, as shown in Figure 3.

Taking one of the target pixels as the target window and setting the maximum ship length to 300 meters, the calculation method of $D_{1}$ is as follows:

$$
D_{1}=1.5 \times L_{P}\left(L_{P}=\frac{300}{R}\right)
$$

where $R$ represents the resolution and $L_{p}$ represents the maximum length of the pixel points of the ship.

$D_{2}$ is calculated as follows: 


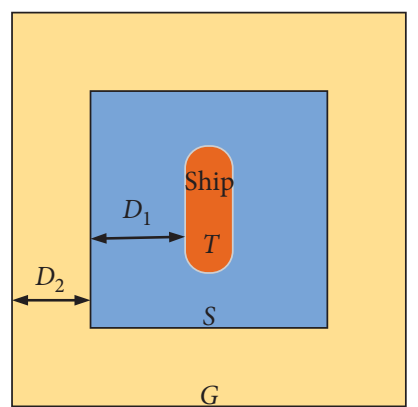

FIgURE 3: Window of global target detection.

$D_{2}= \begin{cases}\frac{\sqrt{\left(2 D_{1}+1\right)^{2}+20000}-\left(2 D_{1}+1\right)}{2}, & R \leq 15 \mathrm{~m}, \\ \frac{\sqrt{\left(2 D_{1}+1\right)^{2}+1000}-\left(2 D_{1}+1\right)}{2}, & R>15 \mathrm{~m} .\end{cases}$

3.2. DBM-SVM Model. DBN is a network structure composed of multilayer restricted Boltzmann machine (RBM) in a certain order. The Boltzmann machine (BM) is an undirected graph model composed of two layers of neural network structures, where the connections within the layers are simultaneous with those between the layers. The structures of BM and RBM are shown in Figure 4.

$\mathrm{RBM}$ is an energy-based network structure consisting of a display layer and an implicit layer. Gibbs sampling is not efficient, while contrast divergence (CD) algorithm [27-29] can reconstruct the data. The main idea of CD is to introduce training data as initial values and conduct Gibbs sampling. The transferred data are taken as the initialization data of the second iteration so that the expectation of quickly solving the model itself is realized. Since the number of iterations is not large, the process of the partial derivative of the likelihood function to the parameters can be simplified to a large extent.

The RBM parameters are updated as follows:

$$
\begin{aligned}
\Delta W_{i j} & =\varepsilon\left(\left\langle v_{i} h_{j}\right\rangle_{\mathrm{data}}-\left\langle v_{i} h_{j}\right\rangle_{l}\right), \\
\Delta a_{i} & =\varepsilon\left(\left\langle v_{i}\right\rangle_{\mathrm{data}}-\left\langle v_{i}\right\rangle_{l}\right), \\
\Delta b_{j} & =\varepsilon\left(\left\langle h_{j}\right\rangle_{\mathrm{data}}-\left\langle h_{j}\right\rangle_{l}\right),
\end{aligned}
$$

where $\varepsilon$ is the learning rate and $l$ represents the data of the reconstruction layer.

If the display layer in RBM contains $n$ nodes and the hidden layer contains $m$ nodes, the steps of CD algorithm are as follows:

(1) It is assumed that the display layer offset is 0.01 , the hidden layer offset is 0.01 , and the initial weight is 0.0008 in the initial parameters

(2) Calculate the probability of each node of hidden layer $h_{1}$ by displaying layer node data $v_{1}$ :

$$
P\left(h_{1 j}=1 \mid v_{1}\right)=\sigma\left(b_{j}+\sum_{i} v_{1 i} W_{i j}\right) .
$$

(3) After obtaining the distribution probability of the hidden layer $h_{1}$, the probability of each node of the next display layer $v_{2}$ can be calculated:

$$
P\left(v_{2 i}=1 \mid h_{1}\right)=\sigma\left(a_{i}+\sum_{j} W_{i j} h_{1 j}\right) .
$$

(4) Using the obtained node probability of display layer $v_{2}$, calculate the node probability of next hidden layer $h_{2}$ :

$$
P\left(h_{2 j}=1 \mid v_{2}\right)=\sigma\left(b_{j}+\sum_{i} v_{2 i} W_{i j}\right) .
$$

(5) Update parameters and repeat steps 2 to 5 until the maximum iteration times are reached, thereby completing the training of an RBM. The RBM algorithm flow is shown in Figure 5.

$\mathrm{DBN}$ is an unsupervised algorithm for feature extraction. During the training, if the error increases greatly, the accuracy of the model may become worse. In order to obtain a robust network structure, supervised callback is adopted to optimize the weights. By using SVM as the classifier in the last classification layer of DBN, the supervised fine-tune optimization of weights is realized. The structure of DBNSVM is shown in Figure 6.

3.3. Target Detection Combining Global CFAR and DBN. The global CFAR detection algorithm greatly improves the detection speed of SAR images without reducing the detection accuracy, but the accuracy is lower than that of DBNSVM algorithm. DBN-SVM has a strong image recognition ability, but the detection speed is slow. In view of this, this paper combines the respective advantages of the two algorithms and proposes a ship detection method in SAR images combining global CFAR and DBN.

The ship small target detection steps based on CFARDBN are as follows:

(1) Training a DBN-SVM network model and obtaining training and test samples, construct a DBN-SVM optimal network model with a small enough error rate through the samples and save the model.

(2) Performing global detection on the whole SAR image, the histogram distribution is used to carry out simple statistics on that gray value of all the pixels. Set the CFAR value to obtain the global threshold, quickly screening out all possible target points and obtaining a target index matrix.

(3) Performing local detection by using the trained DBN-SVM optimal model and taking the pixels screened out in the global detection as the center, set test samples with a size of $90 \times 90$ and substitute 


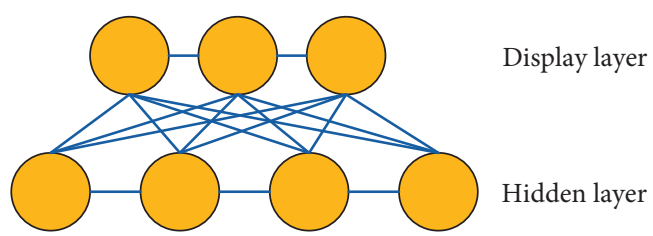

(a)

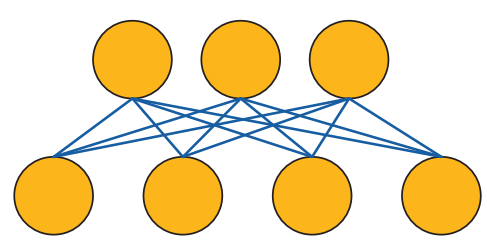

(b)

Figure 4: Structure of BM and RBM. (a) BM. (b) RBM.

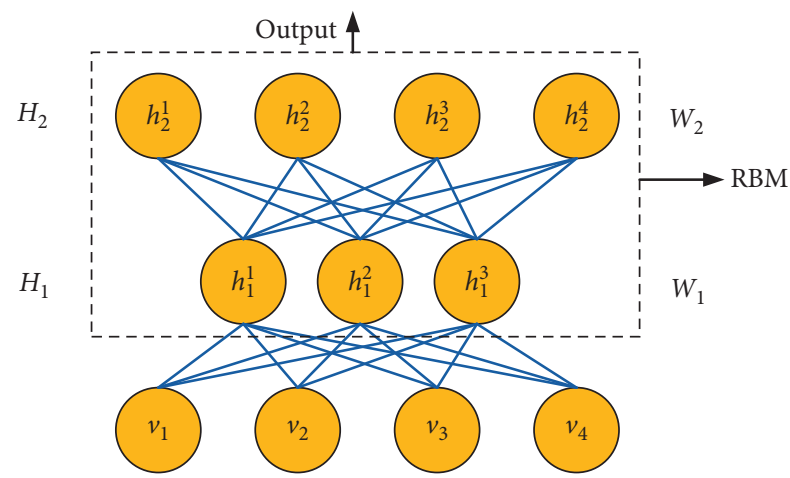

FIGURE 5: RBM algorithm flowchart.

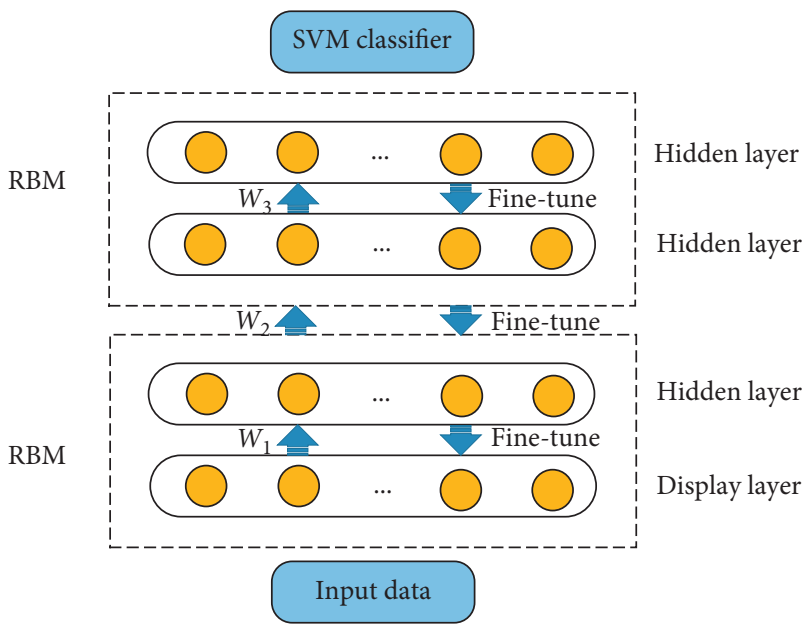

FIGURE 6: Structure of DBN-SVM.

these samples into the trained DBN-SVM optimal network model to obtain the classification results of ships and sea clutter.

\section{Experiment and Result Analysis}

4.1. Experimental Environment and Dataset. The processor of the computer used in the experiment is Intel (R) Core (TM) i5-9400 CPU @ $2.90 \mathrm{GHz} 2.90 \mathrm{GHz}$, the graphics card is NVIDIA GeForce GTX1660 (6 GB), the memory size is $16 \mathrm{~GB}$, the simulation environment is MATLAB R2017a, and the SVM classifier uses LIBSVM toolbox. The DBN training method uses DeepLearnToolbox.

There are 43,819 SAR images with a size of $256 \times 256$ in the small target ship dataset $[30,31]$ used in the experiment, all of which are from GF-3 satellite and Sentinel-1 satellite with corresponding tag information. In this experiment, the ratio of the training set to the test set is $7: 3$. Experiments of fuzzy jamming in different proportions are carried out on SAR images in the test set.

In the fuzzy jamming experiment for each SAR image, two images are superimposed on the principle of the same size and similar pixel size to generate jamming images separately. Some of the jamming images are shown in Figure 7.

4.2. SAR Image Detection Results. Training datasets are trained under the traditional CFAR [12], BPNN [15], Faster R-CNN [16], SSD512 [17], and CFAR-DBN algorithms. After the corresponding training model is obtained, the test dataset is tested under the above five algorithms. The schematic diagram of CFAR-DBN algorithm results display in this paper is shown in Figure 8.

From the visual detection results of some SAR image slices, it can be seen that all algorithms can detect the ships in the original SAR image well under the same conditions. However, after adding different fuzzy interference backgrounds, each algorithm is different in visualization performance. Among them, CFAR-DBN algorithm can well detect all ships under different fuzzy interference conditions. This shows that the adaptability of CFAR-DBN algorithm to fuzzy interference background has been greatly improved, and the applicability is higher, which can meet the needs of complex marine environment monitoring.

In order to study the robustness of the proposed algorithms, it is also necessary to obtain the average precision value of each algorithm. Average precision is an important performance index of target detection in deep learning algorithm. The average accuracy of each algorithm in the interference background is shown in Table 1. The detection time of each algorithm in interference background is shown in Table 2.

It can be seen from Table 1 that the average accuracy of CFAR-DBN algorithm is obviously higher than that of other four algorithms. Under four kinds of fuzzy disturbances, the average accuracy of CFAR-DBN algorithm is higher than $80 \%$. It can be seen from Table 2 that although the traditional CFAR algorithm has the shortest running time, its accuracy is the worst. SSD512 algorithm has the slowest detection speed. The proposed CFAR-DBN algorithm not only ensures high detection accuracy but also improves the detection speed. 


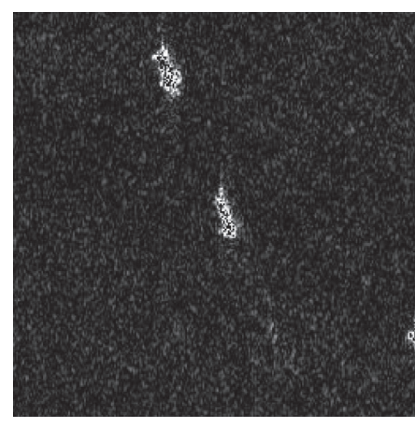

original SAR image

(a)

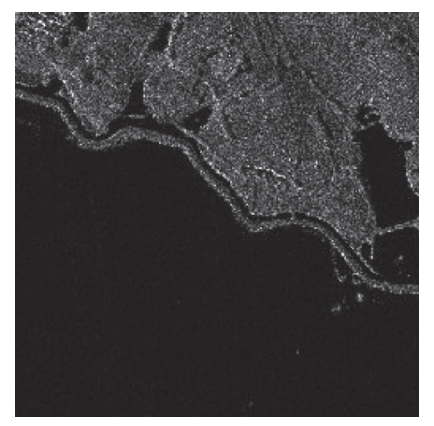

Fuzzy background a

(b)

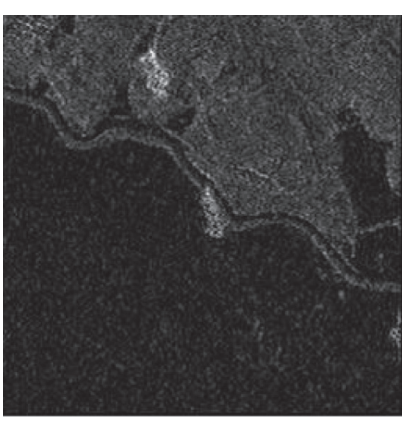

Fuzzy generative graph a

(c)

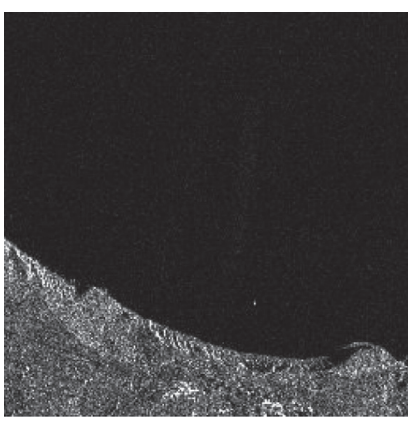

Fuzzy background b

(d)

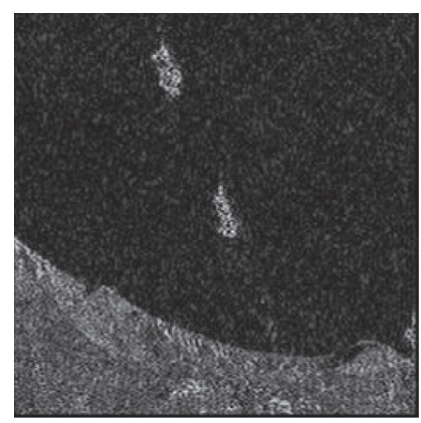

Fuzzy generative graph b

(e)

FIGURE 7: Schematic diagram of ambiguity interference. (a) Original SAR image. (b) Fuzzy background a. (c) Fuzzy generative graph a. (d) Fuzzy background b. (e) Fuzzy generative graph b.

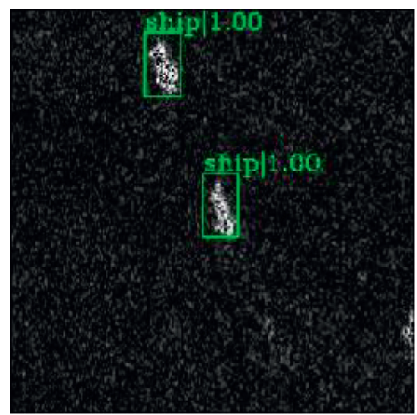

original SAR image

(a)

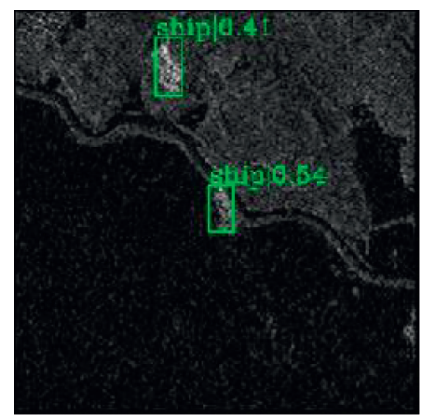

Fuzzy generative graph a

(b)

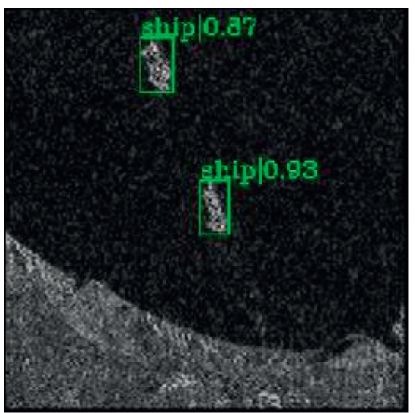

Fuzzy generative graph b

(c)

FIGURE 8: Schematic diagram of CFAR-DBN visual result. (a) Original SAR image. (b) Fuzzy generative graph a. (c) Fuzzy generative graph b.

TABLE 1: Average precision value (\%) of each algorithm under interference background.

\begin{tabular}{lccccc}
\hline Background & Traditional CFAR & BPNN & Faster R-CNN & SSD512 & CFAR-DBN \\
\hline Original background & 87.67 & 87.81 & 88.92 & 90.34 & 91.06 \\
Fuzzy generative graph a & 81.24 & 82.81 & 83.54 & 82.06 & 85.31 \\
Fuzzy generative graph b & 80.82 & 81.25 & 78.27 & 84.63 \\
Fuzzy generative graph c & 77.23 & 78.09 & 89.64 & 80.06 \\
Fuzzy generative graph d & 83.40 & 83.14 & 84.85 & 85.17 & 86.24 \\
\hline
\end{tabular}


TABLE 2: Detection time (s) of each algorithm under interference background.

\begin{tabular}{lccccc}
\hline Background & Traditional CFAR & BPNN & Faster R-CNN & SSD512 & CFAR-DBN \\
\hline Original background & 36 & 58 & 64 & 72 & 64 \\
Fuzzy generative graph a & 41 & 62 & 71 & 102 & 68 \\
Fuzzy generative graph b & 39 & 60 & 82 & 115 & 65 \\
Fuzzy generative graph c & 49 & 71 & 83 & 76 \\
Fuzzy generative graph d & 52 & 74 & 122 & 89 \\
\hline
\end{tabular}

\section{Conclusions}

This paper attempts to combine CFAR algorithm with DBN to solve the problem of uneven background (fuzzy interference) of clutter interference. Firstly, the global detection in CFAR algorithm is used to obtain the target index matrix. Then, taking the target index as the center, the detection image is intercepted, and the DBN model that has been trained in DBN-SVM algorithm is used to detect it. Experimental results show that the proposed DBN-SVM algorithm overcomes the influence of fuzzy interference on small target detection of ships on the sea surface and improves the accuracy and robustness of target detection. At present, the network model of deep learning is large and has a large number of parameters, so it is difficult to deploy quickly and achieve an efficient and accurate detection level in actual SAR image ship target detection. Therefore, the lightweight of the model is the focus of future research.

\section{Data Availability}

The experimental data used to support the findings of this study are available from the corresponding author upon request.

\section{Conflicts of Interest}

The authors declare that they have no conflicts of interest to report regarding the present study.

\section{References}

[1] M. Barber, F. Grings, and C. Bruscantini, "Rationale behind an optimal field experiment to assess the suitability of soil moisture retrieval algorithms for SAR data," IEEE Geoscience and Remote Sensing Letters, vol. 12, no. 4, pp. 791-795, 2017.

[2] O. Yuzugullu, E. Erten, and I. Hajnsek, "Rice growth monitoring by means of X-band co-polar SAR: feature clustering and BBCH scale," IEEE Geoscience and Remote Sensing Letters, vol. 12, no. 6, pp. 1218-1222, 2017.

[3] J. Yang, J. Wang, and R. Lin, "The first quantitative remote sensing of ocean internal waves by Chinese GF-3 SAR satellite," Acta Oceanologica Sinica, vol. 36, no. 01, p. 118, 2017.

[4] H. Zhang and R. Xu, "Exploring the optimal integration levels between SAR and optical data for better urban land cover mapping in the pearl river delta," International Journal of Applied Earth Observation and Geoinformation, vol. 64, pp. 87-95, 2018.

[5] Y. Bai, B. Adriano, B. Adriano, E. Mas, H. Gokon, and S. Koshimura, "Object-based building damage assessment methodology using only post event ALOS-2/PALSAR-2 dual polarimetric SAR intensity images," Journal of Disaster Research, vol. 12, no. 2, pp. 259-271, 2017.

[6] C. Dubois, A. Nascetti, A. Thiele, M. Crespi, and S. Hinz, "SAR-SIFT for matching multiple SAR images and radargrammetry," PFG-Journal of Photogrammetry, Remote Sensing and Geoinformation Science, vol. 85, no. 3, pp. 149158, 2017.

[7] H. Bu, R. Tao, and X. Bai, "A novel SAR imaging algorithm based on compressed sensing," IEEE Geoscience and Remote Sensing Letters, vol. 12, no. 5, pp. 1003-1007, 2017.

[8] S. V. Baumgartner and G. Krieger, "Simultaneous high-resolution wide-swath SAR imaging and ground moving target indication: processing approaches and system concepts," Ieee Journal of Selected Topics in Applied Earth Observations and Remote Sensing, vol. 8, no. 11, pp. 5015-5029, 2017.

[9] B. Huang, Y. Li, and X. Han, "Cloud removal from optical satellite imagery with SAR imagery using sparse representation," IEEE Geoscience and Remote Sensing Letters, vol. 12, no. 5, pp. 1046-1050, 2017.

[10] P. Iervolino, R. Guida, and P. Whittaker, "A model for the backscattering from a canonical ship in SAR imagery," Ieee Journal of Selected Topics in Applied Earth Observations and Remote Sensing, vol. 9, no. 3, pp. 1163-1175, 2017.

[11] T. Sivasankar, S. Ghosh, and M. Joshi, "Exploitation of optical and SAR amplitude imagery for landslide identification: a case study from Sikkim, northeast India," Environmental Monitoring and Assessment, vol. 193, no. 7, pp. 1-17, 2021.

[12] D. Tao, A. P. Doulgeris, and C. Brekke, "A segmentationbased CFAR detection algorithm using truncated statistics," IEEE Transactions on Geoscience and Remote Sensing, vol. 54, no. 5, pp. 2887-2898, 2016.

[13] R. Palamà, M. S. Greco, and F. Gini, "Multistatic adaptive CFAR detection in non-Gaussian clutter," Journal on Advances in Signal Processing, vol. 16, no. 1, pp. 23-34, 2016.

[14] W. Jiang, Y. Huang, and J. Yang, "Automatic censoring CFAR detector based on ordered data difference for low-flying helicopter safety," Sensors, vol. 16, no. 7, pp. 57-69, 2016.

[15] M. A. Rahman, M. S. Hossain, N. A. Alrajeh, and N. Guizani, "B5G and explainable deep learning assisted healthcare vertical at the edge: COVID-I9 perspective," IEEE Network, vol. 34, no. 4, pp. 98-105, 2020.

[16] M. Zreik, R. W. van Hamersvelt, N. Khalili et al., "Deep learning analysis of coronary arteries in cardiac CT angiography for detection of patients requiring invasive coronary angiography," IEEE Transactions on Medical Imaging, vol. 39, no. 5, pp. 1545-1557, 2020.

[17] D. Gao, E. Morini, and M. Salani, “A deep learning approach to identify gene targets of a therapeutic for human splicing disorders," Nature Communications, vol. 12, no. 1, pp. 11-19, 2021.

[18] J. L. Wang, X. Q. Lv, and M. Zhang, "Ship detection in remote sensing images based on improved R-FCN," Progress in Laser and Optoelectronics, vol. 56, no. 16, pp. 214-220, 2019. 
[19] Z. Chen, D. Chen, and Y. Zhang, "Deep learning for autonomous ship-oriented small ship detection," Safety Science, vol. 130, pp. 104-112, 2020.

[20] H. Zhang, Y. Li, Z. Lv, A. K. Sangaiah, and T. Huang, "A realtime and ubiquitous network attack detection based on deep belief network and support vector machine," IEEE/CAA Journal of Automatica Sinica, vol. 7, no. 3, pp. 790-799, 2020.

[21] C. Zhang, Q. W. Gong, and K. Koyamada, "Visual analytics and prediction system based on deep belief networks for icing monitoring data of overhead power transmission lines," Journal of Visualization, vol. 23, no. 6, pp. 1-14, 2020.

[22] J. Wang, J. Liu, P. Ren, and C.-X. Qin, "A SAR target recognition based on guided reconstruction and weighted normconstrained deep belief network," IEEE Access, vol. 8, pp. 181712-181722, 2020.

[23] K. J. Mohler, B. J. Bohn, and Y. Ming, "Dual-comb coherent Raman spectroscopy with lasers of $1 \mathrm{GHz}$ pulse repetition frequency," Optics Letters, vol. 42, no. 2, pp. 318-321, 2016.

[24] A. Rey and C. Delrieux, "Multithresholding techniques in SAR image classification," Remote Sensing Applications Society and Environment, vol. 23, no. 2, pp. 15-23, 2021.

[25] S. Clerc, P. Rieu, and T. Moreau, "Exploiting the sentinel-3 tandem phase dataset and azimuth oversampling to better characterize the sensitivity of SAR altimeter sea surface height to long ocean waves," Advances in Space Research, vol. 67, no. 1, pp. 128-137, 2020.

[26] A. Bentamy, A. Mouche, A. Grouazel, A. Moujane, and A. A. Mohamed, "Using sentinel-1A SAR wind retrievals for enhancing scatterometer and radiometer regional wind analyses," International Journal of Remote Sensing, vol. 40, no. 3, pp. 1120-1147, 2019.

[27] Le Sun, Q. Yu, D. Peng, S. Subramani, and X. Wang, "Fogmed: a fog-based framework for disease prognosis based medical sensor data streams," Computers, Materials \& Continua, vol. 66, no. 1, pp. 603-619, 2021.

[28] Y. Wang, Le Sun, and S. Subramani, "CAB: classifying arrhythmias based on imbalanced sensor data," KSII Transactions on Internet \& Information Systems, vol. 15, no. 7, pp. p2304-2320, 2021.

[29] E. R. Merino, F. M. Castrillejo, and J. D. Pin, "Weighted contrastive divergence," Neural Networks, vol. 106, pp. 147156, 2018.

[30] X. Wang and C. Chen, "Ship detection for complex background SAR images based on a multiscale variance weighted image entropy method," IEEE Geoscience and Remote Sensing Letters, vol. 16, no. 2, pp. 1-4, 2017.

[31] S. Hao, Q. Zhang, and M. Bian, "A novel ship detection method based on gradient and integral feature for singlepolarization synthetic aperture radar imagery," Sensors, vol. 18, no. 2, pp. 563-572, 2018. 